\title{
CRónICAS
}

\section{Reunión del Comité de Jefes de Servicios de Medicina, duRante el XXX Congreso de Medicina INTERNa de la Sociedad Médica de SaNtiago - SOCIEdAd ChIIENA de Medicina INTERNa}

En el XXX Congreso de Medicina Interna realizado recientemente en Coquimbo-La Serena, el 23 y 24 de octubre de 2008, tuvo lugar la reunión del Comité de Jefes de Servicios de Medicina Interna de los diversos centros asistenciales concurrentes.

$\mathrm{Si}$ bien el número de asistentes no sobrepasó 40 personas, los temas presentados relativos al "Médico Hospitalista" y la "Situación del Servicio de Medicina del Hospital San Juan de Dios de Santiago" tuvieron gran realce, dando lugar a un debate y acuerdos aglutinadores de opinión, tales como la necesidad de reforzar este Comité y hacer reuniones similares en el futuro.

Durante esta reunión se hizo evidente que prácticamente todos los Servicios del área pública sufren de serios problemas relativos al recurso camas de hospitalizados, falencias de especialistas y servicios de apoyo, en particular imagenología, laboratorio clínico y cuidados críticos, llegando en algunos casos a niveles de difícil funcionamiento.

Se mencionó que el número de camas del país se ha reducido. En 1996 el país contaba con 29.004 camas, correspondiendo a 3,4 camas por 1.000 habitantes y en el año 2006 existían 26.228 camas, significando un descenso de esta relación a 2,3 camas por 1.000 habitantes, cifra muy por debajo de 4 por 1.000 habitantes, estándar recomendado internacionalmente.

Es un hecho, además, que la atención primaria es deficiente, que los Servicios de Urgencia no dan abasto, manteniendo la misma estructura de corte quirúrgico del pasado, remitiendo pacientes a los Servicios de Medicina con estudios y terapias insatisfactorias. Otras variables analizadas se refirieron al aumento de la población, envejecimiento de los pacientes ingresados a los hospitales, con una mediana de edad de 70 años, con frecuente polipatología y mayor gravedad, donde $20 \%$ po- see un puntaje APACHE sobre 15 puntos, además del "efecto fermento" del GES.

Finalmente se estimó que las disposiciones adoptadas de trabajo en red, reasignación de camas, de camas indiferenciadas o de agudos, constituyan medidas paliativas que no resolverán los problemas de fondo anteriormente comentados, siendo necesario llevar a cabo una transformación real en la administración superior, que involucre la desaparición de los cargos políticos, respetando las jerarquías técnicas.

\section{Dr. Félix Muñoz Cantó Dr. Rodrigo Ponce de Luca}

\section{Curso: Salud en las Américas}

El curso "Salud en las Américas" es un proyecto conjunto entre la Organización Panamericana de la Salud (OPS) y el Departamento de Salud Global de la Universidad del Sur de la Florida (USF).

Este curso aborda el tema de la salud como un derecho humano y examina los factores determinantes fundamentales de la salud y las disparidades que persisten en la Región, para lo cual evaluará la situación de salud actual y sus tendencias en las Américas; examinará los sistemas y programas de atención de salud en la Región; proporcionará un método sistemático para el análisis de la situación de salud en las Américas, con un enfoque en las estadísticas de la Región.

Fechas del curso: 11 al 29 de mayo de 2009; costo: US\$3.500 (incluye libros, materiales del curso, alojamiento y comida). Prerrequisitos: grado universitario en un campo relacionado con la salud, otorgado por una universidad reconocida. Fecha límite de aplicación: 15 de enero de 2009. El formulario de inscripción lo puede descargar en el enlace: http://publications.paho.org

Para más información: Comunicarse con la Dra. Patricia L Ruiz (ruizpatr@paho.org) o visite la dirección: http://publications.paho.org 